\title{
The newest twists in protostellar outflows: triggered star formation
}

\author{
Mary Barsony $\dagger$ \\ ${ }^{1}$ Space Science Institute, 4750 Walnut St., Suite 205, Boulder, CO 80301, USA \\ email: barsony@spacescience.org
}

\begin{abstract}
Winds and outflows powered by the release of gravitational potential energy of infalling matter have been invoked as the triggering agent for further star-formation on scales from dwarf galaxies to individual, low-mass stars. This brief review will touch upon the circumstances under which outflows can serve as star-formation triggers, from specific observations of present-day, nearby protostellar flows.
\end{abstract}

Keywords. circumstellar matter, stars: formation, stars: winds, outflows, ISM: jets and outflows

\section{Observational manifestations of outflows}

Protostellar outflows are recognized as such by the radiative cooling of shocked interstellar gas they generate. Since their discovery thirty years ago (Zuckerman et al. 1976), bipolar outflows generated by forming stars have been observed via millimeter emission lines of tracer molecules, such as CO, CS, SiO (e.g., Bally \& Lada (1983), Fukui et al. (1993), Bachiller (1996), Wolf-Chase et al. (1998), Chandler et al. (2001), Hirano et al. (2006)) and molecular ions such as $\mathrm{HCO}^{+}$(Aso et al. (2000), Rawlings et al. (2004), Arce \& Sargent (2006)). At optical wavelengths, the most commonly observed outflow tracers used in imaging studies are the emission lines of the [SII] doublet at $6716 \AA$ and $6731 \AA$ and of H $\alpha$ at $6563 \AA$ (Mundt \& Fried (1983), Bally et al. (1997), Reipurth \& Bally (2001)). The $2.122 \mu \mathrm{m} 1-0 \mathrm{~S}(1)$ transition of the $\mathrm{H}_{2}$ molecule has been used to trace outflow shocks for three decades (Gautier et al. 1976). Dramatic improvements in areal coverage and sensitivity of the narrowband $\mathrm{H}_{2}$ images have been attained with the relatively recent advent of large-format near-infrared detectors, such as in the WIRC instrument at Palomar, IRIS2 at the AAO, ISPI at CTIO, and WFCAM on UKIRT.

The most recent addition to the arsenal of outflow-imaging techniques has been the IRAC on-board the Spitzer Space Telescope. Of IRAC's four passbands, centered at $3.6 \mu \mathrm{m}$ [Band 1], $4.5 \mu \mathrm{m}$ [Band 2], $5.8 \mu \mathrm{m}$ [Band 3], and $8.0 \mu \mathrm{m}$ [Band 4], the first three contain 6, 7, and $8 \mathrm{H}_{2}$ emission lines, respectively (Smith \& Rosen (2005)).

Band 2 has been an especially useful outflow tracer, since it also encompasses the CO $\mathrm{v}=1-0$ fundamental vibrational emission band at $4.7 \mu \mathrm{m}$, as well as the $\mathrm{HI} \mathrm{Br} \alpha$ emission line at $4.05 \mu \mathrm{m}$.

\section{Observational evidence for outflow-triggered star-formation}

\subsection{The case of $L 1448$}

Figure 1 shows the Spitzer IRAC view of an $11^{\prime} \times 11^{\prime}$ field in Perseus $(\mathrm{d}=350 \mathrm{pc})$ harboring four known Class 0 sources and their outflows. The Class 0 sources are L1448C,

$\dagger$ Present address: San Francisco State University, Department of Physics and Astronomy, 1600 Holloway Drive, San Francisco, CA 94132 USA 


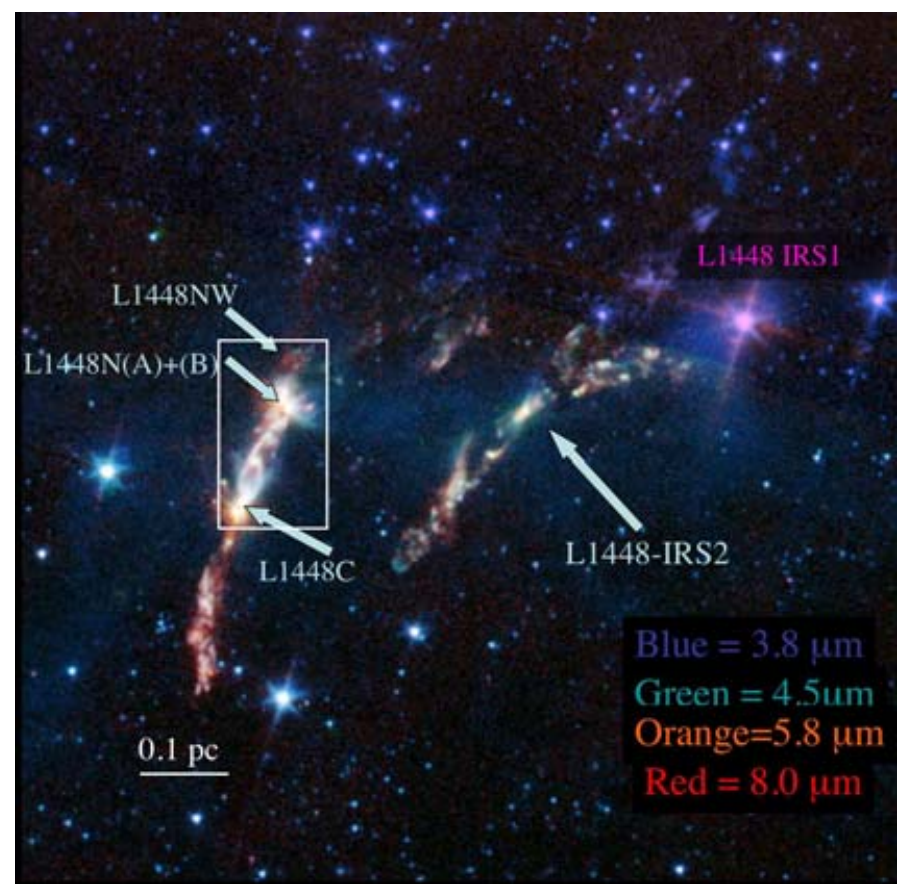

Figure 1. Color-composite image of Spitzer's IRAC view of the $11^{\prime} \times 11^{\prime}$ L1448 field, produced by Dr. Robert Hurt of the Spitzer Science Center. $\mathrm{N}$ is up, and $\mathrm{E}$ is to the left in this image. See text for details.

the $7^{\prime \prime}$ separation proto-binary, L1448N(A) + L1448N(B), and L1448 IRS2. The nature of L1448NW remains to be determined-it may be a pre-stellar clump or another Class 0 protostar. L1448 IRS1 is known to be a more evolved, Class I object. One of the most remarkable features of this multi-band image is the scattered light appearance of the outflow cavity walls carved out by each outflow. A conical, V-shaped, bluish-tinged scattered light structure is seen opening towards the NW from L1448C, towards the W originating from L1448N(B), and exhibiting a bipolar appearance towards L1448 IRS2. Comparison of this image with the published high-velocity blue- and red-shifted CO gas map of the region (Wolf-Chase et al. 2000) reveals the blue-shifted component of the outflow driven by L1448N(B) for the first time. Wolf-Chase et al. had already made the case for the possibility of two flows driven by L1448 IRS2, based on the morphology of the blue-shifted high-velocity CO gas in the vicinity. However, the additional information provided by Figure 1, that the scattered light cavity of L1448N(B) points towards the West, calls for a revised interpretation. The shock structures that seem to emanate to the NW from L1448 IRS2, bending in a southerly direction near L1448 IRS1, and are coincident with high-velocity blue-shifted $\mathrm{CO}$, are actually all manifestations of the blueshifted lobe of the L1448N(B) flow, identified here for the first time. Since L1448 IRS2 has been claimed to be relatively the youngest of these four Class 0 objects (O'Linger et al. 2006), there is the possibility that the collision of the blue-shifted flow driven by L1448N(B) may have triggered the collapse of the pre-stellar clump that formed L1448 IRS2.

However, for a clear observational case for outflow-induced star-formation, we refer the reader to Figure 2, which is an enlarged view of the region outlined by the light-blue rectangle surrounding the protostars, L1448C and L1448N(A)+(B), in Figure 1. In Figure 2 , red dots indicate the positions of the millimeter continuum sources in this region: 
the protostars, L1448C, L1448N(A), L1448N(B), and the pre-stellar clump/protostellar candidate, L1448NW. The blue-shifted, conical outflow cavities dominated by the scattered light from Band 1 (and, therefore, having a light-blue hue in this image) have been outlined by dark-blue lines, pointing to the NW from $\mathrm{L} 1448 \mathrm{C}$ and to the $\mathrm{W}$ from L1448N(B). The similar structure for L1448N(A), although not as clear, is indicated by the dashed dark blue curve to the NW of L1448N(A). The red-shifted flow/cavity boundaries have been indicated only for L1448C (traced by the solid red curve to the SE), and for $\mathrm{L} 1448 \mathrm{~N}(\mathrm{~A})$ (traced by the dashed red curve towards the SE). The directions of the red-shifted flows powered by L1448C and L1448N(A) are known from other previously published works (Bachiller et al. 1995, Barsony et al. 1998). The positions of $\mathrm{H}_{2}$ knots, identified from ground-based imaging observations, are indicated by letters and arrows. The light blue arrows, pointing towards features labeled U-Z, show the locations of lowexcitation $\mathrm{H}_{2}$ shocks, associated with the red-shifted outflow lobe driven by $\mathrm{L} 1448 \mathrm{~N}(\mathrm{~A})$. The red arrows, pointing towards features A-I, mark the locations of high-excitation $\mathrm{H}_{2}$ emission regions, and are associated with the blue-shifted outflow lobe of L1448C (Davis \& Smith 1995). The white, arc-like objects, with their apexes near features A, E, and $\mathrm{G}$, respectively, are pure $\mathrm{H}_{2}$ emission regions, also detected in narrowband imaging from the ground (Davis et al. 1994). The blue-shifted flow driven by L1448C takes a sharp bend (from the high-excitation $\mathrm{H}_{2}$ shock $\mathrm{H}$ to $\mathrm{I}$ ) right where the flow impacts the gas and dust containing L1448N(A)+L1448N(B). Strong shock emission, detected at $100 \mu \mathrm{m}$ in the HIRES-processed IRAS data of this region, coincides with this bend in the flow, further corroborating the heating and compressional effect the flow is having on the gas structure containing the L1448N binary protostellar system (Barsony et al. 1998). This, then, is strong evidence for the L1448C flow triggering the collapse and formation of the binary protostar, L1448N(A)+L1448N(B).

\subsection{Other nearby star-forming regions}

The borderline Class 0/Class I object, L1551NE, lies directly in the red-shifted outflow lobe of its more famous neighbor, the Class I close binary, L1551/IRS5. Both sources are located in the Taurus dark clouds, at 140 pc distance. Combined Nobeyama singletelescope and interferometric maps of various transitions and isopotomers of the CS molecule of the region encompassing L1551NE were acquired by Yokogawa et al. (2003). These authors interpret the combined morphology and velocity structure of the CSemitting gas surrounding the transitional Class 0/Class I object, L1551NE, as evidence for this gas having been swept up, shocked, and dense enough to trigger the formation of L1551NE from the powerful outflow of its neighbor, L1551 IRS5. The fact that L1551 $\mathrm{NE}$ is actually physically inside the outflow cavity produced by the combined flows of the Class I L1551 IRS 5 binary, and that L1551NE is at a younger evolutionary stage than L1551/IRS5, both strongly support the contention of outflow-triggered star-formation in this case.

The nearby (310-320 pc) NGC 1333 star-forming cluster may be unique in the sheer wealth of outflows it exhibits. Its Spitzer composite color image in all of the IRAC bands was shown by several different speakers at this Symposium. The protostar known as NGC 1333 /IRAS 4A is located within the IRAC image. IRAS 4A is known to be dynamically collapsing on the size scale of its protostellar envelope, from detection of red-shifted absorption of molecular lines against its bright, millimeter dust continuum (Di Francesco et al.. 2001). IRAS 4A is not directly detected by IRAC - one sees only a very dark absorption feature at its location. The greenish-tinged sources in the vicinity of IRAS 4A in the color-composite IRAC image are shocks associated with various outflows. Two of these, HL 6 and HL 9, are associated with near-infrared $2.12 \mu \mathrm{m}$ shocked $\mathrm{H}_{2}$ emission first 


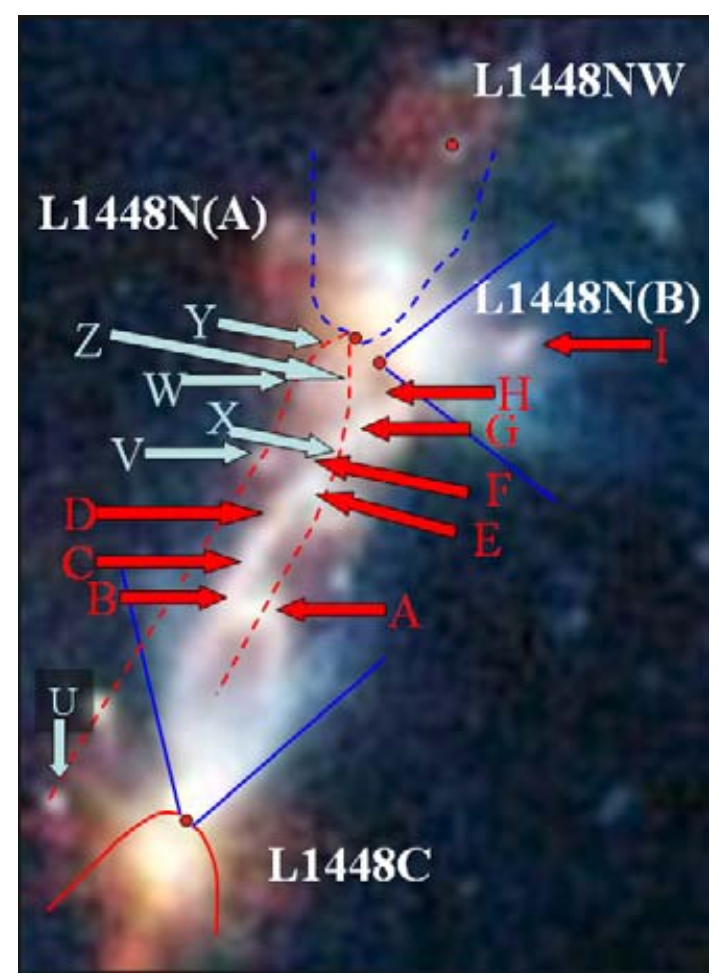

Figure 2. Spitzer's IRAC view of the three Class 0 protostars, L1448C and the wide binary, L1448N(A) and L1448N(B)-all marked by red dots. The dust condensation, L1448NW, also marked by a red dot, may be pre-stellar. North is up and East is to the left in this image. The blue-shifted outflow lobes of each protostar, detected via millimeter $\mathrm{CO}$ emission, are outlined in blue. Red arrows indicate the locations of high-excitation near-infrared $\mathrm{H}_{2}$ shocks, A-I, associated with the blue-shifted portion of the L1448C bipolar flow, whereas light blue arrows indicate the locations of low-excitation near-infrared $\mathrm{H}_{2}$ shocks, $\mathrm{U}-\mathrm{Z}$, associated with the red-shifted lobe of the L1448N(A) bipolar flow. Note that the blue-shifted lobe of the L1448C flow veers sharply to the West-from shock $\mathrm{H}$ to shock I, right at the position of the proto-binary, whose formation this flow may have triggered. Further north, the blue-shifted flow of L1448N(A) may have helped form the pre-stellar condensation, L1448NW.

detected from the ground (Hodapp \& Ladd 1995). The observational evidence for outflowtriggered formation for IRAS $4 \mathrm{~A}$ is not yet conclusive. A coordinated, multi-wavelength study of both the morphology and the kinematics of the gas and flows in the vicinity of IRAS 4A is required before one could make a well-justified case for outflow-triggered star-formation in this instance.

\section{Theoretical considerations regarding outflow-triggered star-formation}

Despite the lack of conclusive observational evidence for the triggering of the collapse of IRAS 4A by a single, identifiable outflow, as was the case for L1551NE and L1448N, IRAS 4A has been modelled as having collapsed due to triggering from a sudden increase in external pressure (Belloche et al. 2006, and article by Hennebelle in this Volume). This sudden pressure increase was assumed to be caused by the combined flows of NGC 1333. A simple non-rotating, spherical Bonnor-Ebert sphere source model was used as a starting point for the SPH code calculation. The initial model is assumed to be embedded 
in a hot rarefied external medium. In order to reproduce the envelope's density structure (as inferred from multi-scale mm continuum observations) and velocity structure (derived from multi-line and isotopic spectral line maps), the applied external pressure to IRAS $4 \mathrm{~A}$ must have doubled in just 3000 years for an envelope with a $10^{5} \mathrm{yr}$. crossing time, in order to be consistent with the densities and velocities deduced from the observations. The resulting model can match the observations over the $1500 \mathrm{AU}-10^{4}$ AU spatial scale for 10,000 to 30,000 yr after the formation of a central "point" mass. The radial mass distributions calculated from the model are too rarefied to reproduce the high densities observed on spatial scales from a few hundred AU to 1500 AU, however. The primary argument for invoking triggering for the collapse of IRAS 4A is its deduced high mass infall rate of $10^{-4} \mathrm{M}_{\odot} \mathrm{yr}^{-1}$, which is 2 orders of magnitude above that expected for a collapsing isothermal sphere.

\section{Outstanding problems and future work}

Outflow triggered star formation is operative on many scales, from intergalactic (e.g., Silk \& Rees 1998), to interstellar, to individual stellar systems. Due to space considerations, only the smallest scales were considered here. A single protostellar flow impinging on an ambient interstellar clump may or may not cause it to collapse and form another protostar. Only the clearest cases of outflow-induced star-formation were presented. There are many instances where outflows impact clumps, but these clumps just remain as obstacles in/to the flow, without collapsing. Future systematic studies of known outflows may distinguish the precise range of conditions under which outflow-triggered star formation occurs.

\section{Acknowledgements}

Helpful discussions with Josh Walawender in comparing multi-waveband images of the Perseus molecular cloud are gratefully acknowledged. M.B. wishes to acknowledge NASA/JPL Spitzer Cycle 3 funding which made her travel to attend the IAU XXV GA in Prague, and this Symposium 237, possible. Parts of this work were also supported under NSF Grants 95-01788, 97-31797, and 02-06146.

\section{References}

Arce, H.G. \& Sargent, A.I. 2006, ApJ 646, 1070

Aso, Y., Tatematsu, K., Sekimoto, Y., Nakano, T., Umemoto, T., Koyama, K. \& Yamamoto, S. 2000, ApJS 131, 465

Bachiller, R., Guilloteau, S., Dutrey, A., Planesas, P. \& Martin-Pintado, J. 1995, A\&GA 299, 857

Bachiller, R. 1996, ARAESA 34, p. 111

Bally, J. \& Lada, C.J. 1983, ApJ 265, 824

Bally, J., Devine, D., Alten, V. \& Sutherland, R.S. 1997, ApJ 478, 603

Barsony, M., Ward-Thompson, D., André, P. \& O'Linger, J. 1998, ApJ 509, 733

Belloche, A., Hennebelle, P. \& André, P. 2006, A\& A 453, 145

Chandler, C.J. \& Richer, J.S. 2001, ApJ 555, 139

Davis, C.J., Dent, W.R.F., Matthews, H.E., Aspin, C. \& Lightfoot, J.F. 1994, MNRAS 266, 933

Davis, C.J. \& Smith, M.D. 1995, ApJ 443, L41

Di Francesco, J., Myers, P.C., Wilner, D.J., Ohashi, N. \& Mardones, D. 2001, ApJ 562, 770

Fukui, Y., Iwata, T., Mizuno, A., Bally, J. \& Lane, A.P. 1993,in E.H. Levy \& J.I. Lunine (eds.), Protostars 83 Planets III (Tucson: Univ. of Arizona), p. 603

Gautier, T.N., Fink, U., Larson, H.P. \& Treffers, R.R. 1976, ApJ 207, L129

Hirano, N., Liu, S.-Y., Shang, H., Ho, P.T.P., Huang, H.-C., Kuan, Y.-J., McCaughrean, M.J. \& Zhang, Q. 2006, ApJ 636, L141 
Hodapp, K.-W. \& Ladd, E.F. 1995, ApJ 453, 715

Mundt, R. \& Fried, J.W. 1983, ApJ 274, L83

O'Linger, J.C., Cole, D.M., Ressler, M.E. \& Wolf-Chase, G. 2006, AJ 131, 2601

Reipurth, B. \& Bally, J. 2001, ARA\&A 39, p. 403

Rawlings, J.M.C., Redman, M.P., Keto, E. \& Williams, D.A. 2004, MNRAS 351, 1054

Silk, J. \& Rees, M. 1998, A\&\&A 331, L1

Smith, M.D. \& Rosen, A. 2005, MNRAS 357, 1370

Wolf-Chase, G.A., Barsony, M., Wootten, H.A., Ward-Thompson, D., Lowrance, P.J., Kastner, J.H. \& McMullin, J.P. 1998, ApJ 501, L193

Wolf-Chase, G.A., Barsony, M. \& O'Linger, J.. 2000, AJ 120, 1467

Yokogawa, S., Kitamura, Y., Momose, M. \& Kawabe, R. 2003, ApJ 595, 266

Zuckerman, B., Kuiper, T.B.H. \& Rodriguez-Kuiper, E.N. 1976, ApJ 209, L137 